\title{
NON-SELF-ADJOINT FOURTH-ORDER DISSIPATIVE OPERATORS AND THE COMPLETENESS OF THEIR EIGENFUNCTIONS
}

\author{
Mei-Chun YAng, Ji-Jun Ao And ChaO Li
}

Abstract. A class of non-self-adjoint fourth order differential operators with general separated boundary conditions in Weyl's limit circle case is studied. The dissipation property of the considered operators in $L^{2}[a, b)$ is proven by analysis and by using the characteristic determinant, the completeness of the system of eigenfunctions and associated functions of these dissipative operators also be proven.

Mathematics subject classification (2010): Primary 34L10, 34B20; Secondary 47A48.

Keywords and phrases: Fourth order dissipative operators, eigenvalues, eigenfunctions, completeness, characteristic determinant.

\section{REFERENCES}

[1] I. C. Gohberg, M. G. Krein, Introduction to the Theory of Linear Non-Self-Adjoint Operator, in: Transl. Math. Monographs, vol. 18, American Math. Soc., Providence, RI, 1969.

[2] M. V. KeLDYSH, On the completeness of the eigenfunctions of some classes of non-self-adjoint linear operators, Soviet Math. Dokl. 77 (1951) 11-14.

[3] I. M. Glazman, Direct Methods of Qualitative Spectral Analysis of Singular Differential Operators, Israel Program for Scientific Translations, Jerusalem, 1965.

[4] A. R. Sims, Secondary conditions for linear differential operators of the second order, J. Math. Mech. 6 (1957) 247-285.

[5] V. A. MARCHENKO, Expansion in eigenfunctions of non-self-adjoint singular second order differential operators, American Math. Soc. Transl. Ser. 225 (1963) 77-139.

[6] M. A. NAimark, Linear Differential Operators, Ungar, New York, 1968.

[7] Z. WANG, H. Wu, Dissipative non-self-adjoint Sturm-Liouville operators and completeness of their eigenfunctions, J. Math. Anal. and Appl. 394 (2012) 1-12.

[8] G. Sh. Guseinov, H. Tuncay, The determinants of perturbation connected with a dissipative Sturm-liouville operators, J. Math. Anal. and Appl. 194 (1995) 39-49.

[9] E. Bairamov, A. M. Krall, Dissipative operators generated by the Sturm-Liouville differential expression in the Weyl limit circle case, J. Math. Anal. and Appl. 254 (2001) 178-190.

[10] E. Bairamov, E. Ugurlu, The determinants of dissipative Sturm-Liouville operators with transmission conditions, Math. and Computer Modelling 53 (2011) 805-813.

[11] E. Ugurlu, E. Bairamov, On Singular Dissipative Fourth-Order Differential Operator in Lim-4 Case, ISRN Math. Anal. 2013 (2013) article ID 549876, 5 pages.

[12] X. Y. ZHANG, J. SUn, The determinants of fourth order dissipative operators with transmission conditions, J. Math. Anal. and Appl. 410 (2014) 55-69.

[13] E. Ugurlu, E. BAIRAMov, On the rate of the convergence of the characteristic values of an integral operator associated with a dissipative fourth order differential operator in lim-4 case with finite transmission conditions, J. Math. Chem. 52 (2014) 2627-2644.

[14] H. TunA, On spectral properties of dissipative fourth order boundary-value problem with a spectral parameter in the boundary condition, Appl. Math. Comput. 219 (2013) 9377-9387.

[15] A. WAng, J. SUn AND A. ZettL, The classification of self-adjoint boundary conditions: Separated, coupled, and mixed, J. Funct. Analysis 255 (2008) 1554-1573. 
[16] X. HaO, J. SUn AND A. ZetTL, Canonical forms of self-adjoint boundary conditions for differential operators of order four, J. Math. Anal. and Appl. 387 (2012) 1176-1187.

[17] Z. CAO, Ordinary Differential Operators, Shanghai Science and Technology Press, Shanghai, 1987 (in chinese).

[18] M. S. P. Eastham, The lim-2n case of symmetric differential operators of order $2 n$, Pro. London Math. Soc. 38 (3) (1979) 272-294.

[19] W. N. Everitt, I. W. Knowles, T. T. Read, Limit-point and limit-circle criteria for Sturmliouville equations with intermittently negative principal coefficient, Pro. Roy. Soc. Edinburgh Sect. A 103 (1986) 215-228.

[20] B. J. HARRIS, Limit-circle criteria for second order differential expression, Quart. J. Math. Oxford 35 (2) (1984) 415-427.

[21] A. ZettL, Sturm-Liouville Theory, Amer. Math. Soc., Mathematical Surveys and Monographs 121, Providence, RI, 2005.

[22] M. G. KREIN, On the indeterminant case of the Sturm-liouville boundary problems in the interval $(0,+\infty)$, Lzv. Akad. Nuak SSSR Ser. Mat. 16 (1952) 293-324. 